\title{
EXISTENCE OF POSITIVE PERIODIC SOLUTION \\ OF A PERIODIC COOPERATIVE MODEL WITH \\ DELAYS AND IMPULSES
}

YONGKUN LI AND WENYA XING

Received 1 December 2005; Accepted 2 May 2006

Sufficient conditions are obtained for the existence of at least one positive periodic solution of a periodic cooperative model with delays and impulses by using Mawhin's continuation theorem of coincidence degree theory.

Copyright (c) 2006 Hindawi Publishing Corporation. All rights reserved.

\section{Introduction}

In 1974 May [10] suggested the following cooperative species model [3]:

$$
\begin{aligned}
& \dot{x}_{1}(t)=r_{1} x_{1}(t)\left[1-\frac{x_{1}(t)}{a_{1}+b_{1} x_{2}(t)}-c_{1} x_{1}(t)\right], \\
& \dot{x}_{2}(t)=r_{2} x_{2}(t)\left[1-\frac{x_{2}(t)}{a_{2}+b_{2} x_{1}(t)}-c_{2} x_{2}(t)\right],
\end{aligned}
$$

where $a_{i}, b_{i}$, and $c_{i}, i=1,2$, are positive constants. Recently, paper [11] has studied the existence of positive periodic solutions of the following system:

$$
\begin{aligned}
& \dot{x}_{1}(t)=r_{1}(t) x_{1}(t)\left[1-\frac{x_{1}(t)}{a_{1}(t)+b_{1}(t) x_{2}(t)}-c_{1}(t) x_{1}(t)\right], \\
& \dot{x}_{2}(t)=r_{2}(t) x_{2}(t)\left[1-\frac{x_{2}(t)}{a_{2}(t)+b_{2}(t) x_{1}(t)}-c_{2}(t) x_{2}(t)\right],
\end{aligned}
$$

where $a_{i}, b_{i}$, and $c_{i}(i=1,2)$ are nonnegative $\omega$-periodic continuous functions. It is well known that more realistic and interesting species models should take into account both the seasonality of the changing environment and time delays $[4,8,9]$, and that the birth of many species is not continuous but occurs at fixed time intervals (some wild animals have seasonal births), in the long run; the birth of these species can be considered as an impulse to the system $[1,2,5,7]$. To describe this phenomenon exactly, we proposed 
2 Positive periodic solution of a cooperative model

the following periodic cooperative species model with delays and impulses, which is a generalization of (1.1) and (1.2),

$$
\begin{gathered}
\frac{\mathrm{d} x_{1}(t)}{\mathrm{d} t}=r_{1}(t) x_{1}(t)\left[1-\frac{x_{1}\left(t-\tau_{11}(t)\right)}{a_{1}(t)+b_{1}(t) x_{2}\left(t-\tau_{12}(t)\right)}-c_{1}(t) x_{1}\left(t-\tau_{13}(t)\right)\right], \quad t>0, t \neq t_{k}, \\
\Delta x_{1}\left(t_{k}\right)=-\gamma_{1 k} x_{1}\left(t_{k}\right), \quad k=1,2, \ldots, \\
\frac{\mathrm{d} x_{2}(t)}{\mathrm{d} t}=r_{2}(t) x_{2}(t)\left[1-\frac{x_{2}\left(t-\tau_{21}(t)\right)}{a_{2}(t)+b_{2}(t) x_{1}\left(t-\tau_{22}(t)\right)}-c_{2}(t) x_{2}\left(t-\tau_{23}(t)\right)\right], \quad t>0, t \neq t_{k}, \\
\Delta x_{2}\left(t_{k}\right)=-\gamma_{2 k} x_{2}\left(t_{k}\right), \quad k=1,2, \ldots,
\end{gathered}
$$

where $\Delta x\left(t_{k}\right)=x\left(t_{k}^{+}\right)-x\left(t_{k}^{-}\right)$are the impulses at moment $t_{k}$ and $t_{1}<t_{2}<\cdots$ is a strictly increasing sequence such that $\lim _{k \rightarrow \infty} t_{k}=+\infty$ and there exists a positive integer $q$ such that $t_{k+q}=t_{k}+\omega, \gamma_{i(k+q)}=\gamma_{i k}<1, k=1,2, \ldots, i=1,2, r_{i}(t), a_{i}(t), c_{i}(t), i=1,2$, are positive continuous $\omega$-periodic functions, $b_{i}(t), \tau_{i j}(t), i=1,2, j=1,2,3$, are nonnegative continuous $\omega$-periodic functions.

As usual in the theory of impulsive differential equations, at the points of discontinuity $t_{k}$ of the solution $t \mapsto x_{i}(t)$ we assume that $x_{i}\left(t_{k}\right) \equiv x_{i}\left(t_{k}^{-}\right)$. It is clear that, in general, the derivatives $x_{i}^{\prime}\left(t_{k}\right)$ do not exist. On the other hand, according to the first equality (1.3) there exist the limits $x_{i}^{\prime}\left(t_{k}^{\mp}\right)$. According to the above convention, we assume $x_{i}^{\prime}\left(t_{k}\right) \equiv x_{i}^{\prime}\left(t_{k}^{-}\right)$.

Throughout this paper, we assume that

$$
\prod_{r_{\sigma} \leq t_{k}<t}\left(1-\gamma_{i k}\right), \quad i=1,2
$$

are $\omega$-periodic functions.

The organization of this paper is as follows. In Section 2, we introduce some notations and definitions, and state some preliminary results needed in later sections. We then study, in Section 3, the existence of periodic solutions of system (1.3) by using the continuation theorem of coincidence degree theory proposed by Gaines and Mawhin [6].

\section{Preliminaries}

In order to obtain the existence of a positive periodic solution of system (1.3), we first make the following preparations.

Consider the impulsive system

$$
\begin{gathered}
x^{\prime}(t)=f\left(t, x(t), x\left(t-\tau_{1}(t)\right), \ldots, x_{n}\left(t-\tau_{n}(t)\right)\right), \quad t \neq t_{k}, k=1,2, \ldots, \\
\left.\Delta x(t)\right|_{t=t_{k}}=I_{k}\left(x\left(t_{k}^{-}\right)\right),
\end{gathered}
$$


where $x \in \mathbb{R}^{n}, f: \mathbb{R} \times \mathbb{R}^{n+1} \rightarrow \mathbb{R}^{n}$ is continuous, and $f$ is $\omega$-periodic with respect to its first argument; $I_{k}: \mathbb{R}^{n} \rightarrow \mathbb{R}^{n}$ are continuous, and there exists a positive integer $q$ such that $t_{k+q}=t_{k}+\omega, I_{k+q}(x)=I_{k}(x)$ with $t_{k} \in \mathbb{R}, t_{k+1}>t_{k}, \lim _{k \rightarrow \infty} t_{k}=\infty,\left.\Delta x(t)\right|_{t=t_{k}}=x\left(t_{k}^{+}\right)-$ $x\left(t_{k}^{-}\right)$. For $t_{k} \neq 0(k=1,2, \ldots),[0, \omega] \cap\left\{t_{k}\right\}=\left\{t_{1}, t_{2}, \ldots, t_{q}\right\}$. As we know, $\left\{t_{k}\right\}$ are called points of jump.

For any $\sigma \geq t_{0}$, let

$$
r_{\sigma}=\min _{1 \leq i \leq n} \inf _{t \geq \sigma}\left\{t-\tau_{i}(t)\right\}
$$

and let $P C_{\sigma}$ denote the set of functions $\phi:\left[r_{\sigma}, \sigma\right] \rightarrow \mathbb{R}$ which are real-valued absolutely continuous in $\left[t_{k}, t_{k+1}\right) \cap\left(r_{\sigma}, \sigma\right)$ and at $t_{k}$ situated in $\left(r_{\sigma}, \sigma\right]$ may have discontinuity of the first kind.

Definition 2.1. For any $\sigma \geq 0$ and $\phi \in P C_{\sigma}$, a function $x \in\left(\left[r_{\sigma}, \infty\right), \mathbb{R}\right)$ denoted by $x(t, \sigma, \phi)$ is said to be a solution of system $(2.1)$ on $(\sigma, \infty]$ satisfying the initial value conditions

$$
x(t)=\phi(t), \quad \phi(\sigma)>0, \quad t \in\left[r_{\sigma}, \sigma\right]
$$

if the following conditions are satisfied:

(i) $x(t)$ is absolutely continuous on each interval $\left(t_{k}, t_{k+1}\right) \subset\left[r_{\sigma}, \infty\right)$;

(ii) for any $t_{k} \in[\sigma, \infty), k=1,2, \ldots, x\left(t_{k}^{+}\right)$and $x\left(t_{k}^{-}\right)$exist and $x\left(t_{k}^{-}\right)=x\left(t_{k}\right)$;

(iii) $x(t)$ satisfies $(2.1)$ for almost everywhere in $[\sigma, \infty)$ and at impulsive points $t_{k}$ situated in $[\sigma, \infty)$ may have discontinuity of the first kind.

Consider the following nonimpulsive delay differential system

$$
\begin{aligned}
& \frac{\mathrm{d} y_{1}(t)}{\mathrm{d} t}=r_{1}(t) y_{1}(t)\left[1-\frac{\prod_{0 \leq t_{k}<t-\tau_{11}(t)}\left(1-\gamma_{1 k}\right) y_{1}\left(t-\tau_{11}(t)\right)}{a_{1}(t)+b_{1}(t) \prod_{0 \leq t_{k}<t-\tau_{12}(t)}\left(1-\gamma_{2 k}\right) y_{2}\left(t-\tau_{12}(t)\right)}\right. \\
& \left.-c_{1}(t) \prod_{0 \leq t_{k}<t-\tau_{13}(t)}\left(1-\gamma_{1 k}\right) y_{1}\left(t-\tau_{13}(t)\right)\right] \\
& \frac{\mathrm{d} y_{2}(t)}{\mathrm{d} t}=r_{2}(t) y_{2}(t)\left[1-\frac{\prod_{0 \leq t_{k}<t-\tau_{21}(t)}\left(1-\gamma_{2 k}\right) y_{2}\left(t-\tau_{21}(t)\right)}{a_{2}(t)+b_{2}(t) \prod_{0 \leq t_{k}<t-\tau_{22}(t)}\left(1-\gamma_{1 k}\right) y_{1}\left(t-\tau_{22}(t)\right)}\right. \\
& \left.-c_{2}(t) \prod_{0 \leq t_{k}<t-\tau_{23}(t)}\left(1-\gamma_{2 k}\right) y_{2}\left(t-\tau_{23}(t)\right)\right] \text {, }
\end{aligned}
$$

with initial condition $y_{i}(t)=\phi_{i}(t), t \leq 0$, where $\phi_{i}(t)$ is defined as above.

In the following, we will establish a fundamental theorem that enables us to reduce the existence of solution of system (1.3) to the corresponding problem for the nonimpulsive delay differential system (2.4). 


\section{Positive periodic solution of a cooperative model}

Theorem 2.2. Assume that (1.4) holds. Then

(i) if $y=\left(y_{1}, y_{2}\right)^{T}$ is a solution of (2.4), then

$$
x=\left(\prod_{0 \leq t_{k}<t}\left(1-\gamma_{1 k}\right) y_{1}, \prod_{0 \leq t_{k}<t}\left(1-\gamma_{2 k}\right) y_{2}\right)^{T}
$$

is a solution of (1.3);

(ii) if $x=\left(x_{1}, x_{2}\right)^{T}$ is a solution of (1.3), then

$$
y=\left(\prod_{0 \leq t_{k}<t}\left(1-\gamma_{1 k}\right)^{-1} x_{1}, \prod_{0 \leq t_{k}<t} \ln \left(1-\gamma_{2 k}\right)^{-1} x_{2}\right)^{T}
$$

is a solution of (2.4).

Proof. First, we prove (i). It is easy to see that $x_{i}=\prod_{0 \leq t_{k}<t}\left(1-\gamma_{i k}\right) y_{i}, i=1,2$, are absolutely continuous on the interval $\left(t_{k}, t_{k+1}\right]$ and that for any $t \neq t_{k}, k=1,2 \ldots$,

$$
x=\left(\prod_{0 \leq t_{k}<t}\left(1-\gamma_{1 k}\right) y_{1}, \prod_{0 \leq t_{k}<t}\left(1-\gamma_{2 k}\right) y_{2}\right)^{T}
$$

satisfies system (1.3).

On the other hand, for every $t_{k} \in\left\{t_{k}\right\}$,

$$
\begin{gathered}
x_{i}\left(t_{k}^{+}\right)=\lim _{t \rightarrow t_{k}^{+}} \prod_{0 \leq t_{j}<t}\left(1-\gamma_{i j}\right) y_{i}(t)=\prod_{0 \leq t_{j} \leq t_{k}}\left(1-\gamma_{i j}\right) y_{i}\left(t_{k}\right), \quad i=1,2, \\
x_{i}\left(t_{k}\right)=\prod_{0 \leq t_{j}<t_{k}}\left(1-\gamma_{i j}\right) y_{i}\left(t_{k}\right), \quad i=1,2 .
\end{gathered}
$$

Thus, for every $k=1,2, \ldots$,

$$
x_{i}\left(t_{k}^{+}\right)=\left(1-\gamma_{i k}\right) x_{i}\left(t_{k}\right), \quad i=1,2 .
$$

The proof is complete.

Next, we prove (ii). Since $x_{i}(t)$ is absolutely continuous on each interval $\left(t_{k}, t_{k+1}\right]$ and, in view of (2.9), it follows that, for any $k=1,2, \ldots$,

$$
\begin{gathered}
y_{i}\left(t_{k}^{+}\right)=\prod_{0 \leq t_{j} \leq t_{k}}\left(1-\gamma_{i j}\right)^{-1} x_{i}\left(t_{k}^{+}\right)=\prod_{0 \leq t_{j}<t_{k}}\left(1-\gamma_{i j}\right)^{-1} x_{i}\left(t_{k}\right)=y_{i}\left(t_{k}\right), \quad i=1,2, \\
y_{i}\left(t_{k}^{-}\right)=\prod_{0 \leq t_{j} \leq t_{k-1}}\left(1-\gamma_{i j}\right)^{-1} x_{i}\left(t_{k}^{-}\right)=y_{i}\left(t_{k}\right), \quad k=1,2, \ldots,
\end{gathered}
$$

which implies that $y_{i}(t), i=1,2$, are continuous on $[0, \infty)$. It is easy to prove that $y_{i}(t)$ are absolutely continuous on $[0, \infty)$. Now, one can easily check that

$$
y=\left(\prod_{0 \leq t_{k}<t}\left(1-\gamma_{1 k}\right)^{-1} x_{1}, \prod_{0 \leq t_{k}<t}\left(1-\gamma_{2 k}\right)^{-1} x_{2}\right)^{T}
$$

is a solution of (2.9). The proof is complete. 


\section{Existence of periodic solutions}

In this section, based on Mawhin's continuation theorem, we will study the existence of at least one periodic solution of (1.3). To do so, we will make some preparations.

Let $\mathbb{X}$ and $\mathbb{Y}$ be real Banach spaces, $L: \operatorname{Dom} L \subset \mathbb{X} \rightarrow \mathbb{Y}$ a linear mapping, and $N$ : $\mathbb{X} \rightarrow \mathbb{Y}$ a continuous mapping. The mapping $L$ will be called a Fredholm mapping of index zero if $\operatorname{dim} \operatorname{Ker} L=\operatorname{codim} \operatorname{Im} L<+\infty$ and $\operatorname{Im} L$ is closed in $\mathbb{Y}$. If $L$ is a Fredholm mapping of index zero and there exist continuous projectors $P: \mathbb{X} \rightarrow \mathbb{X}$ and $Q: \mathbb{Y} \rightarrow \mathbb{Y}$ such that $\operatorname{Im} P=\operatorname{Ker} L$ and $\operatorname{Ker} Q=\operatorname{Im}(I-Q)$, it follows that mapping $\left.L\right|_{\operatorname{Dom} L \cap \operatorname{Ker} P}:(I-$ $P) \mathbb{X} \rightarrow \operatorname{Im} L$ is invertible. We denote the inverse of that mapping by $K_{P}$. If $\Omega$ is an open bounded subset of $\mathbb{X}$, the mapping $N$ will be called $L$-compact on $\bar{\Omega}$ if $Q N(\bar{\Omega})$ is bounded and $K_{P}(I-Q) N: \bar{\Omega} \rightarrow \mathbb{X}$ is compact. Since $\operatorname{Im} Q$ is isomorphic to $\operatorname{Ker} L$, there exists an isomorphism $J: \operatorname{Im} Q \rightarrow \operatorname{Ker} L$.

Now, we introduce Mawhin's continuation theorem [6, page 40] as follows.

Lemma 3.1. Let $\Omega \subset \mathbb{X}$ be an open bounded set and let $N: \mathbb{X} \rightarrow \mathbb{Y}$ be a continuous operator which is L-compact on $\bar{\Omega}$. Assume

(a) for each $\lambda \in(0,1), x \in \partial \Omega \cap \operatorname{Dom} L, L x \neq \lambda N x$;

(b) for each $x \in \partial \Omega \cap \operatorname{Ker} L, Q N x \neq 0$, and $\operatorname{deg}(J Q N, \Omega \cap \operatorname{Ker} L, 0) \neq 0$.

Then $L x=N x$ has at least one solution in $\bar{\Omega} \cap \operatorname{Dom} L$.

In what follows, we will use the following notations:

$$
\bar{h}=\frac{1}{\omega} \int_{0}^{\omega} h(t) \mathrm{d} t, \quad h^{m}=\min _{t \in[0, \omega]}\{h(t)\}, \quad h^{M}=\max _{t \in[0, \omega]}\{h(t)\},
$$

where $h(t)$ is a periodic continuous function with period $\omega$,

$$
\begin{array}{ll}
A_{1}^{s}=\sup _{t \in[0, \omega]}\left\{\prod_{0 \leq t_{k}<t}\left[\ln \left(1-\gamma_{1 k}\right)\right]^{-1}\right\}, & B_{1}^{s}=\sup _{t \in[0, \omega]}\left\{\prod_{0 \leq t_{k}<t}\left[\ln \left(1-\gamma_{2 k}\right)\right]^{-1}\right\}, \\
A_{1}^{f}=\inf _{t \in[0, \omega]}\left\{\prod_{0 \leq t_{k}<t}\left[\ln \left(1-\gamma_{1 k}\right)\right]^{-1}\right\}, & B_{1}^{f}=\inf _{t \in[0, \omega]}\left\{\prod_{0 \leq t_{k}<t}\left[\ln \left(1-\gamma_{2 k}\right)\right]^{-1}\right\}, \\
A_{2}^{s}=\sup _{t \in[0, \omega]}\left\{\prod_{0 \leq t_{k}<t} \ln \left(1-\gamma_{1 k}\right)\right\}, & B_{2}^{s}=\sup _{t \in[0, \omega]}\left\{\prod_{0 \leq t_{k}<t} \ln \left(1-\gamma_{2 k}\right)\right\}, \\
A_{2}^{f}=\inf _{t \in[0, \omega]}\left\{\prod_{0 \leq t_{k}<t} \ln \left(1-\gamma_{1 k}\right)\right\}, & B_{2}^{f}=\inf _{t \in[0, \omega]}\left\{\prod_{0 \leq t_{k}<t} \ln \left(1-\gamma_{2 k}\right)\right\} .
\end{array}
$$

Before we proceed to state and prove our main result, we introduce a lemma which is useful in the proof of our main result.

Let

$$
y_{1}(t)=\exp \left\{z_{1}(t)\right\}, \quad y_{2}(t)=\exp \left\{z_{2}(t)\right\},
$$


6 Positive periodic solution of a cooperative model

then (2.4) is transformed into

$$
\begin{aligned}
\frac{\mathrm{d} z_{1}(t)}{\mathrm{d} t}=r_{1}(t)[ & 1-\frac{\prod_{0 \leq t_{k}<t-\tau_{11}(t)}\left(1-\gamma_{1 k}\right) \exp \left\{z_{1}\left(t-\tau_{11}(t)\right)\right\}}{a_{1}(t)+b_{1}(t) \prod_{0 \leq t_{k}<t-\tau_{12}(t)}\left(1-\gamma_{2 k}\right) \exp \left\{z_{2}\left(t-\tau_{12}(t)\right)\right\}} \\
& \left.-c_{1}(t) \prod_{0 \leq t_{k}<t-\tau_{13}(t)}\left(1-\gamma_{1 k}\right) \exp \left\{z_{1}\left(t-\tau_{13}(t)\right)\right\}\right], \\
\frac{\mathrm{d} z_{2}(t)}{\mathrm{d} t}=r_{2}(t)[ & 1-\frac{\prod_{0 \leq t_{k}<t-\tau_{21}(t)}\left(1-\gamma_{2 k}\right) \exp \left\{z_{2}\left(t-\tau_{21}(t)\right)\right\}}{a_{2}(t)+b_{2}(t) \prod_{0 \leq t_{k}<t-\tau_{22}(t)}\left(1-\gamma_{1 k}\right) \exp \left\{z_{1}\left(t-\tau_{22}(t)\right)\right\}} \\
& \left.-c_{2}(t) \prod_{0 \leq t_{k}<t-\tau_{23}(t)}\left(1-\gamma_{2 k}\right) \exp \left\{z_{2}\left(t-\tau_{23}(t)\right)\right\}\right] .
\end{aligned}
$$

One can easily check that if system (3.4) has an $\omega$-periodic solution $\left(z_{1}^{*}(t), z_{2}^{*}(t)\right)^{T}$, then $\left(e^{z_{1}^{*}(t)}, e^{z_{2}^{*}(t)}\right)^{T}$ is a positive $\omega$-periodic solution of system $(2.4)$.

LEMMA 3.2. Let

$$
\begin{aligned}
f\left(z_{1}, z_{2}\right)=\left(\bar{r}_{1}-\frac{1}{\omega} \int_{0}^{\omega} \frac{r_{1}(t) \prod_{0 \leq t_{k}<t-\tau_{11}(t)}\left(1-\gamma_{1 k}\right) \exp \left\{z_{1}\right\}}{a_{1}(t)+b_{1}(t) \prod_{0 \leq t_{k}<t-\tau_{12}(t)}\left(1-\gamma_{2 k}\right) \exp \left\{z_{2}\right\}} \mathrm{d} t\right. \\
\quad-\frac{1}{\omega} \int_{0}^{\omega} c_{1}(t) r_{1}(t) \prod_{0 \leq t_{k}<t-\tau_{13}(t)}\left(1-\gamma_{1 k}\right) \exp \left\{z_{1}\right\} \mathrm{d} t, \\
\\
\bar{r}_{2}-\frac{1}{\omega} \int_{0}^{\omega} \frac{r_{2}(t) \prod_{0 \leq t_{k}<t-\tau_{21}(t)}\left(1-\gamma_{2 k}\right) \exp \left\{z_{2}\right\}}{a_{2}(t)+b_{2}(t) \prod_{0 \leq t_{k}<t-\tau_{22}(t)}\left(1-\gamma_{1 k}\right) \exp \left\{z_{1}\right\}} \mathrm{d} t \\
\left.\quad-\frac{1}{\omega} \int_{0}^{\omega} c_{2}(t) r_{2}(t) \prod_{0 \leq t_{k}<t-\tau_{23}(t)}\left(1-\gamma_{2 k}\right) \exp \left\{z_{2}\right\} \mathrm{d} t\right)^{T},
\end{aligned}
$$

and $\Omega=\left\{\left(z_{1}, z_{2}\right)^{T} \in \mathbb{R}^{2}:\left\|\left(z_{1}, z_{2}\right)^{T}\right\|<H_{0}\right\}$, where $r_{i}, a_{i}, b_{i}, c_{i}, i=1,2$, are the same as those in system (1.3) and

$$
\begin{aligned}
& H_{0}>\max \left\{\left|\ln \frac{\bar{r}_{1}}{c_{1}^{m} r_{1}^{m} A_{2}^{f}}\right|,\left|\ln \frac{\bar{r}_{2}}{c_{2}^{m} r_{2}^{m} B_{2}^{f}}\right|,\left|\ln \frac{\bar{r}_{1}}{\left[\left(a_{1}^{m}\right)^{-1}+c_{1}^{M}\right] r_{1}^{M} A_{2}^{s}}\right|,\right. \\
&\left.\left|\ln \frac{\bar{r}_{2}}{\left[\left(a_{2}^{m}\right)^{-1}+c_{2}^{M}\right] r_{2}^{M} B_{2}^{s}}\right|\right\}
\end{aligned}
$$

is a constant. Then

$$
\operatorname{deg}\{f, \Omega,(0,0)\} \neq 0 .
$$


Proof. Set

$$
\begin{aligned}
\Phi\left(z_{1}, z_{2}, \delta\right)=\left(\bar{r}_{1}-\frac{1}{\omega} \int_{0}^{\omega} \frac{r_{1}(t) \prod_{0 \leq t_{k}<t-\tau_{11}(t)}\left(1-\gamma_{1 k}\right) \exp \left\{z_{1}\right\}}{a_{1}(t)+\delta b_{1}(t) \prod_{0 \leq t_{k}<t-\tau_{12}(t)}\left(1-\gamma_{2 k}\right) \exp \left\{z_{2}\right\}} \mathrm{d} t\right. \\
-\frac{1}{\omega} \int_{0}^{\omega} c_{1}(t) r_{1}(t) \prod_{0 \leq t_{k}<t-\tau_{13}(t)}\left(1-\gamma_{1 k}\right) \exp \left\{z_{1}\right\} \mathrm{d} t, \\
\bar{r}_{2}-\frac{1}{\omega} \int_{0}^{\omega} \frac{r_{2}(t) \prod_{0 \leq t_{k}<t-\tau_{21}(t)}\left(1-\gamma_{2 k}\right) \exp \left\{z_{2}\right\}}{a_{2}(t)+\delta b_{2}(t) \prod_{0 \leq t_{k}<t-\tau_{22}(t)}\left(1-\gamma_{1 k}\right) \exp \left\{z_{1}\right\}} \mathrm{d} t \\
\left.-\frac{1}{\omega} \int_{0}^{\omega} c_{2}(t) r_{2}(t) \prod_{0 \leq t_{k}<t-\tau_{23}(t)}\left(1-\gamma_{2 k}\right) \exp \left\{z_{2}\right\} \mathrm{d} t\right)^{T},
\end{aligned}
$$

then it is easy to see that, for $\left(z_{1}, z_{2}, \delta\right) \in \mathbb{R}^{2} \times[0,1]$,

$$
\begin{aligned}
\bar{r}_{1}- & \frac{1}{\omega} \int_{0}^{\omega} \frac{r_{1}(t) \prod_{0 \leq t_{k}<t-\tau_{11}(t)}\left(1-\gamma_{1 k}\right) \exp \left\{z_{1}\right\}}{a_{1}(t)+\delta b_{1}(t) \prod_{0 \leq t_{k}<t-\tau_{12}(t)}\left(1-\gamma_{2 k}\right) \exp \left\{z_{2}\right\}} \mathrm{d} t \\
- & \frac{1}{\omega} \int_{0}^{\omega} c_{1}(t) r_{1}(t) \prod_{0 \leq t_{k}<t-\tau_{13}(t)}\left(1-\gamma_{1 k}\right) \exp \left\{z_{1}\right\} \mathrm{d} t \\
& <\bar{r}_{1}-\frac{1}{\omega} \int_{0}^{\omega} c_{1}(t) r_{1}(t) \prod_{0 \leq t_{k}<t-\tau_{13}(t)}\left(1-\gamma_{1 k}\right) \exp \left\{z_{1}\right\} \mathrm{d} t \\
& <\bar{r}_{1}-c_{1}^{m} r_{1}^{m} A_{2}^{f} \exp \left\{z_{1}\right\}<0 \quad \text { as } z_{1} \geq \frac{H_{0}}{2}, \\
\bar{r}_{2}- & \frac{1}{\omega} \int_{0}^{\omega} \frac{r_{2}(t) \prod_{0 \leq t_{k}<t-\tau_{21}(t)}\left(1-\gamma_{2 k}\right) \exp \left\{z_{2}\right\}}{a_{2}(t)+\delta b_{2}(t) \prod_{0 \leq t_{k}<t-\tau_{22}(t)}\left(1-\gamma_{1 k}\right) \exp \left\{z_{1}\right\}} \mathrm{d} t \\
- & \frac{1}{\omega} \int_{0}^{\omega} c_{2}(t) r_{2}(t) \quad \prod_{0 \leq t_{k}<t-\tau_{23}(t)}\left(1-\gamma_{2 k}\right) \exp \left\{z_{2}\right\} \mathrm{d} t \\
& <\bar{r}_{2}-\frac{1}{\omega} \int_{0}^{\omega} c_{2}(t) r_{2}(t) \prod_{0 \leq t_{k}<t-\tau_{23}(t)}\left(1-\gamma_{2 k}\right) \exp \left\{z_{2}\right\} \mathrm{d} t \\
& \bar{r}_{2}^{m} r_{2}^{m} B_{2}^{f} \exp \left\{z_{2}\right\}<0 \quad \text { as } z_{2} \geq \frac{H_{0}}{2},
\end{aligned}
$$


8 Positive periodic solution of a cooperative model

$$
\begin{aligned}
& \bar{r}_{1}- \frac{1}{\omega} \int_{0}^{\omega} \frac{r_{1}(t) \prod_{0 \leq t_{k}<t-\tau_{11}(t)}\left(1-\gamma_{1 k}\right) \exp \left\{z_{1}\right\}}{a_{1}(t)+\delta b_{1}(t) \prod_{0 \leq t_{k}<t-\tau_{12}(t)}\left(1-\gamma_{2 k}\right) \exp \left\{z_{2}\right\}} \mathrm{d} t \\
&- \frac{1}{\omega} \int_{0}^{\omega} c_{1}(t) r_{1}(t) \prod_{0 \leq t_{k}<t-\tau_{13}(t)}\left(1-\gamma_{1 k}\right) \exp \left\{z_{1}\right\} \mathrm{d} t \\
& \geq \bar{r}_{1}-\frac{1}{\omega} \int_{0}^{\omega} \frac{r_{1}^{M} A_{2}^{s} \exp \left\{z_{1}\right\}}{a_{1}^{m}} d t-\frac{1}{\omega} \int_{0}^{\omega} c_{1}^{M} r_{1}^{M} A_{2}^{s} \exp \left\{z_{1}\right\} \mathrm{d} t \\
&=\bar{r}_{1}-\left[\left(a_{1}^{m}\right)^{-1}+c_{1}^{M}\right] r_{1}^{M} A_{2}^{s} \exp \left\{z_{1}\right\}>0 \quad \text { as } z_{1} \leq-\frac{H_{0}}{2}, \\
& \bar{r}_{2}-\frac{1}{\omega} \int_{0}^{\omega} \frac{r_{2}(t) \prod_{0 \leq t_{k}<t-\tau_{21}(t)}\left(1-\gamma_{2 k}\right) \exp \left\{z_{2}\right\}}{a_{2}(t)+\delta b_{2}(t) \prod_{0 \leq t_{k}<t-\tau_{22}(t)}\left(1-\gamma_{1 k}\right) \exp \left\{z_{1}\right\}} \mathrm{d} t \\
&-\frac{1}{\omega} \int_{0}^{\omega} c_{2}(t) r_{2}(t) \prod_{0 \leq t_{k}<t-\tau_{23}(t)}\left(1-\gamma_{2 k}\right) \exp \left\{z_{2}\right\} \mathrm{d} t \\
& \quad \geq \bar{r}_{2}-\frac{1}{\omega} \int_{0}^{\omega} \frac{r_{2}^{M} B_{2}^{s} \exp \left\{z_{2}\right\}}{a_{2}^{m}} d t-\frac{1}{\omega} \int_{0}^{\omega} c_{2}^{M} r_{2}^{M} B_{2}^{s} \exp \left\{z_{2}\right\} \mathrm{d} t \\
&=\bar{r}_{2}-\left[\left(a_{2}^{m}\right){ }^{-1}+c_{2}^{M}\right] r_{2}^{M} B_{2}^{s} \exp \left\{z_{2}\right\}>0 \text { as } z_{2} \leq-\frac{H_{0}}{2} .
\end{aligned}
$$

Therefore,

$$
\Phi\left(z_{1}, z_{2}, \delta\right) \neq 0 \quad \text { for }\left(z_{1}, z_{2}, \delta\right) \in \partial \Omega \times[0,1] .
$$

From the property of invariance under a homotopy, we have

$$
\operatorname{deg}\left\{f\left(z_{1}, z_{2}\right), \Omega,(0,0)\right\}=\operatorname{deg}\left\{\Phi\left(z_{1}, z_{2}, 0\right), \Omega,(0,0)\right\} .
$$

By a straightforward computation, we find

$$
\operatorname{deg}\left\{\Phi\left(z_{1}, z_{2}, 0\right), \Omega,(0,0)\right\}=-1 \neq 0 .
$$

This completes the proof.

We are now in a position to state and prove the existence of periodic solutions of (1.3).

Theorem 3.3. Assume that (1.4) holds. Suppose further that

(i) $a_{1}^{m}>A_{2}^{s} \exp \left\{M_{1}\right\}$;

(ii) $a_{2}^{m}>B_{2}^{s} \exp \left\{M_{2}\right\}$;

where $M_{1}=\ln \left(A_{1}^{s} / c_{1}^{m}\right)+2 \omega r_{1}^{M}, M_{2}=\ln \left(B_{1}^{s} / c_{2}^{m}\right)+2 \omega r_{2}^{M}$. Then system (1.3) has at least one positive $\omega$-periodic solution.

Proof. According to the discussion made in Section 2, we need only to prove that the nonimpulsive delay differential system (3.4) has an $\omega$-periodic solution. In order to use 
the continuation theorem of coincidence degree theory to establish the existence of $\omega$ periodic solutions of (3.4), we take $\mathbb{X}=\mathbb{Y}=\left\{\left(z_{1}(t), z_{2}(t)\right)^{T} \in C\left(\mathbb{R}, \mathbb{R}^{2}\right): z_{1}(t+\omega)=z_{1}(t)\right.$, $\left.z_{2}(t+\omega)=z_{2}(t)\right\}$, and $\left\|\left(z_{1}(t), z_{2}(t)\right)^{T}\right\|=\max _{t \in[0, \omega]}\left|z_{1}(t)\right|+\max _{t \in[0, \omega]}\left|z_{2}(t)\right|$. With this norm, $\mathbb{X}$ and $\mathbb{Y}$ are Banach spaces. Set

$$
\begin{gathered}
L\left[\begin{array}{l}
z_{1} \\
z_{2}
\end{array}\right]=\left[\begin{array}{l}
\dot{z_{1}} \\
\dot{z_{2}}
\end{array}\right], \quad P\left[\begin{array}{l}
z_{1} \\
z_{2}
\end{array}\right]=Q\left[\begin{array}{l}
z_{1} \\
z_{2}
\end{array}\right]=\left[\begin{array}{l}
\frac{1}{\omega} \int_{0}^{\omega} z_{1}(t) \mathrm{d} t \\
\frac{1}{\omega} \int_{0}^{\omega} z_{2}(t) \mathrm{d} t
\end{array}\right], \quad\left[\begin{array}{l}
z_{1} \\
z_{2}
\end{array}\right] \in \mathbb{X}, \\
N\left[\begin{array}{l}
z_{1} \\
z_{2}
\end{array}\right]=\left[\begin{array}{l}
l G_{1}\left(t, z_{1}(t), z_{2}(t)\right) \\
G_{2}\left(t, z_{1}(t), z_{2}(t)\right)
\end{array}\right]
\end{gathered}
$$

where

$$
\begin{aligned}
& G_{1}\left(t, z_{1}(t), z_{2}(t)\right)=r_{1}(t)[ 1-\frac{\prod_{0 \leq t_{k}<t-\tau_{11}(t)}\left(1-\gamma_{1 k}\right) \exp \left\{z_{1}\left(t-\tau_{11}(t)\right)\right\}}{a_{1}(t)+b_{1}(t) \prod_{0 \leq t_{k}<t-\tau_{12}(t)}\left(1-\gamma_{2 k}\right) \exp \left\{z_{2}\left(t-\tau_{12}(t)\right)\right\}} \\
&\left.-c_{1}(t) \prod_{0 \leq t_{k}<t-\tau_{13}(t)}\left(1-\gamma_{1 k}\right) \exp \left\{z_{1}\left(t-\tau_{13}(t)\right)\right\}\right], \\
& G_{2}\left(t, z_{1}(t), z_{2}(t)\right)=r_{2}(t)\left[1-\frac{\prod_{0 \leq t_{k}<t-\tau_{21}(t)}\left(1-\gamma_{2 k}\right) \exp \left\{z_{2}\left(t-\tau_{21}(t)\right)\right\}}{a_{2}(t)+b_{2}(t) \prod_{0 \leq t_{k}<t-\tau_{22}(t)}\left(1-\gamma_{1 k}\right) \exp \left\{z_{1}\left(t-\tau_{22}(t)\right)\right\}}\right. \\
&\left.-c_{2}(t) \prod_{0 \leq t_{k}<t-\tau_{23}(t)}\left(1-\gamma_{2 k}\right) \exp \left\{z_{2}\left(t-\tau_{23}(t)\right)\right\}\right] .
\end{aligned}
$$

Obviously $\operatorname{Ker} L=\mathbb{R}^{2}$ and

$$
\operatorname{dim} \operatorname{Ker} L=2=\operatorname{codim} \operatorname{Im} L .
$$

So, $\operatorname{Im} L$ is closed in $\mathbb{X}$ and $L$ is a Fredholm mapping of index zero. It is easy to show that $P$ and $Q$ are continuous projectors such that

$$
\operatorname{Im} P=\operatorname{Ker} L, \quad \operatorname{Ker} Q=\operatorname{Im}(I-Q) .
$$

Furthermore, the generalized inverse (to $L$ ) $K_{P}: \operatorname{Im} L \rightarrow \operatorname{Dom} L \cap \operatorname{Ker} P$ is given by

$$
K_{P}\left[\begin{array}{l}
z_{1} \\
z_{2}
\end{array}\right]=\left[\begin{array}{l}
\int_{0}^{\omega} z_{1}(s) \mathrm{d} s-\frac{1}{\omega} \int_{0}^{\omega} \int_{0}^{t} z_{1}(s) \mathrm{d} s \mathrm{~d} t \\
\int_{0}^{\omega} z_{2}(s) \mathrm{d} s-\frac{1}{\omega} \int_{0}^{\omega} \int_{0}^{t} z_{2}(s) \mathrm{d} s \mathrm{~d} t
\end{array}\right] .
$$


Thus

$$
Q N\left[\begin{array}{l}
z_{1} \\
z_{2}
\end{array}\right]=\left[\begin{array}{l}
\frac{1}{\omega} \int_{0}^{\omega} G_{1}\left(t, z_{1}(t), z_{2}(t)\right) \mathrm{d} t \\
\frac{1}{\omega} \int_{0}^{\omega} G_{2}\left(t, z_{1}(t), z_{2}(t)\right) \mathrm{d} t
\end{array}\right]
$$

hence

$$
\begin{aligned}
& K_{P}(I-Q) N: \mathbb{X} \longrightarrow \mathbb{X}, \\
& K_{P}(I-Q) N\left[\begin{array}{l}
z_{1} \\
z_{2}
\end{array}\right]= {\left[\begin{array}{l}
\int_{0}^{t} G_{1}\left(s, z_{1}(s), z_{2}(s)\right) \mathrm{d} s \\
\int_{0}^{t} G_{2}\left(s, z_{1}(s), z_{2}(s)\right) \mathrm{d} s
\end{array}\right]-\left[\begin{array}{l}
\left.\frac{1}{\omega} \int_{0}^{\omega} \int_{0}^{t} G_{1}\left(s, z_{1}(s), z_{2}(s)\right) \mathrm{d} s \mathrm{~d} t\right] \\
\left.\frac{1}{\omega} \int_{0}^{\omega} \int_{0}^{t} G_{2}\left(s, z_{1}(s), z_{2}(s)\right) \mathrm{d} s \mathrm{~d} t\right] \\
-
\end{array}\right.} \\
&-\left[\begin{array}{l}
\left(\frac{t}{\omega}-\frac{1}{2}\right) \int_{0}^{\omega} G_{1}\left(s, z_{1}(s), z_{2}(s)\right) \mathrm{d} s \\
\left.\left(\frac{t}{\omega}-\frac{1}{2}\right) \int_{0}^{\omega} G_{2}\left(s, z_{1}(s), z_{2}(s)\right) \mathrm{d} s\right]
\end{array}\right.
\end{aligned}
$$

Clearly, $Q N$ and $K_{P}(I-Q) N$ are continuous. Using the Arzela-Ascoli theorem, it is not difficult to show that $K_{P}(I-Q) N$ is compact for any open bounded set $\Omega \subset \mathbb{X}$. Moreover $\mathrm{QN}(\bar{\Omega})$ is bounded. Thus $N$ is $L$-compact on $\Omega$ with any open bounded set $\Omega \subset \mathbb{X}$. Then isomorphism $J$ of $\operatorname{Im} Q$ onto $\operatorname{Ker} L$ can be the identity mapping $\operatorname{since} \operatorname{Im} Q=\operatorname{Ker} L$.

Now we reach the position to search for an appropriate open bounded subset $\Omega$ for the application of the continuation theorem. Corresponding to the operator equation $L x=\lambda N x, \lambda \in(0,1)$, we have

$$
\begin{aligned}
\frac{\mathrm{d} z_{1}(t)}{\mathrm{d} t}=\lambda r_{1}(t)\left[1-\frac{\prod_{0 \leq t_{k}<t-\tau_{11}(t)}\left(1-\gamma_{1 k}\right) \exp \left\{z_{1}\left(t-\tau_{11}(t)\right)\right\}}{a_{1}(t)+b_{1}(t) \prod_{0 \leq t_{k}<t-\tau_{12}(t)}\left(1-\gamma_{2 k}\right) \exp \left\{z_{2}\left(t-\tau_{12}(t)\right)\right\}}\right. & \\
& \left.-c_{1}(t) \prod_{0 \leq t_{k}<t-\tau_{13}(t)}\left(1-\gamma_{1 k}\right) \exp \left\{z_{1}\left(t-\tau_{13}(t)\right)\right\}\right], \\
\frac{\mathrm{d} z_{2}(t)}{\mathrm{d} t}=\lambda r_{2}(t)\left[1-\frac{\prod_{0 \leq t_{k}<t-\tau_{21}(t)}\left(1-\gamma_{2 k}\right) \exp \left\{z_{2}\left(t-\tau_{21}(t)\right)\right\}}{a_{2}(t)+b_{2}(t) \prod_{0 \leq t_{k}<t-\tau_{22}(t)}\left(1-\gamma_{1 k}\right) \exp \left\{z_{1}\left(t-\tau_{22}(t)\right)\right\}}\right. & \\
& \left.-c_{2}(t) \prod_{0 \leq t_{k}<t-\tau_{23}(t)}\left(1-\gamma_{2 k}\right) \exp \left\{z_{2}\left(t-\tau_{23}(t)\right)\right\}\right] .
\end{aligned}
$$


Suppose that $z(t)=\left(z_{1}(t), z_{2}(t)\right)^{T} \in \mathbb{X}$ is a solution of system (3.20) for some $\lambda \in(0,1)$. Integrating (3.20) over the interval $[0, \omega]$, we obtain

$$
\begin{aligned}
& \int_{0}^{\omega} r_{1}(t) \mathrm{d} t=\int_{0}^{\omega} r_{1}(t) {\left[\frac{\prod_{0 \leq t_{k}<t-\tau_{11}(t)}\left(1-\gamma_{1 k}\right) \exp \left\{z_{1}\left(t-\tau_{11}(t)\right)\right\}}{a_{1}(t)+b_{1}(t) \prod_{0 \leq t_{k}<t-\tau_{12}(t)}\left(1-\gamma_{2 k}\right) \exp \left\{z_{2}\left(t-\tau_{12}(t)\right)\right\}}\right.} \\
&\left.+c_{1}(t) \prod_{0 \leq t_{k}<t-\tau_{13}(t)}\left(1-\gamma_{1 k}\right) \exp \left\{z_{1}\left(t-\tau_{13}(t)\right)\right\}\right] \mathrm{d} t, \\
& \int_{0}^{\omega} r_{2}(t) \mathrm{d} t=\int_{0}^{\omega} r_{2}(t)\left[\frac{\prod_{0 \leq t_{k}<t-\tau_{21}(t)}\left(1-\gamma_{2 k}\right) \exp \left\{z_{2}\left(t-\tau_{21}(t)\right)\right\}}{a_{2}(t)+b_{2}(t) \prod_{0 \leq t_{k}<t-\tau_{22}(t)}\left(1-\gamma_{1 k}\right) \exp \left\{z_{1}\left(t-\tau_{22}(t)\right)\right\}}\right. \\
&\left.+c_{2}(t) \prod_{0 \leq t_{k}<t-\tau_{23}(t)}\left(1-\gamma_{2 k}\right) \exp \left\{z_{2}\left(t-\tau_{23}(t)\right)\right\}\right] \mathrm{d} t .
\end{aligned}
$$

From (3.20) and (3.21), we have

$$
\begin{aligned}
& \int_{0}^{\omega}\left|\dot{z}_{1}(t)\right| \mathrm{d} t=\lambda \int_{0}^{\omega} \mid r_{1}(t)\left[1-\frac{\prod_{0 \leq t_{k}<t-\tau_{11}(t)}\left(1-\gamma_{1 k}\right) \exp \left\{z_{1}\left(t-\tau_{11}(t)\right)\right\}}{a_{1}(t)+b_{1}(t) \prod_{0 \leq t_{k}<t-\tau_{12}(t)}\left(1-\gamma_{2 k}\right) \exp \left\{z_{2}\left(t-\tau_{12}(t)\right)\right\}}\right. \\
& \left.-c_{1}(t) \prod_{0 \leq t_{k}<t-\tau_{13}(t)}\left(1-\gamma_{1 k}\right) \exp \left\{z_{1}\left(t-\tau_{13}(t)\right)\right\}\right] \mid \mathrm{d} t \\
& \leq \lambda \int_{0}^{\omega} \mid r_{1}(t)\left[1+\frac{\prod_{0 \leq t_{k}<t-\tau_{11}(t)}\left(1-\gamma_{1 k}\right) \exp \left\{z_{1}\left(t-\tau_{11}(t)\right)\right\}}{a_{1}(t)+b_{1}(t) \prod_{0 \leq t_{k}<t-\tau_{12}(t)}\left(1-\gamma_{2 k}\right) \exp \left\{z_{2}\left(t-\tau_{12}(t)\right)\right\}}\right. \\
& \left.+c_{1}(t) \prod_{0 \leq t_{k}<t-\tau_{13}(t)}\left(1-\gamma_{1 k}\right) \exp \left\{z_{1}\left(t-\tau_{13}(t)\right)\right\}\right] \mid \mathrm{d} t \\
& \leq 2 \int_{0}^{\omega} r_{1}(t) \mathrm{d} t \leq 2 \omega r_{1}^{M}, \\
& \int_{0}^{\omega}\left|\dot{z}_{2}(t)\right| \mathrm{d} t=\lambda \int_{0}^{\omega} \mid r_{2}(t)\left[1-\frac{\prod_{0 \leq t_{k}<t-\tau_{21}(t)}\left(1-\gamma_{2 k}\right) \exp \left\{z_{2}\left(t-\tau_{21}(t)\right)\right\}}{a_{2}(t)+b_{2}(t) \prod_{0 \leq t_{k}<t-\tau_{22}(t)}\left(1-\gamma_{1 k}\right) \exp \left\{z_{1}\left(t-\tau_{22}(t)\right)\right\}}\right. \\
& \left.-c_{2}(t) \prod_{0 \leq t_{k}<t-\tau_{23}(t)}\left(1-\gamma_{2 k}\right) \exp \left\{z_{2}\left(t-\tau_{23}(t)\right)\right\}\right] \mid \mathrm{d} t \\
& \leq \lambda \int_{0}^{\omega} \mid r_{2}(t)\left[1+\frac{\prod_{0 \leq t_{k}<t-\tau_{21}(t)}\left(1-\gamma_{2 k}\right) \exp \left\{z_{2}\left(t-\tau_{21}(t)\right)\right\}}{a_{2}(t)+b_{2}(t) \prod_{0 \leq t_{k}<t-\tau_{22}(t)}\left(1-\gamma_{1 k}\right) \exp \left\{z_{1}\left(t-\tau_{22}(t)\right)\right\}}\right. \\
& \left.+c_{2}(t) \prod_{0 \leq t_{k}<t-\tau_{23}(t)}\left(1-\gamma_{2 k}\right) \exp \left\{z_{2}\left(t-\tau_{23}(t)\right)\right\}\right] \mid \mathrm{d} t \\
& \leq 2 \int_{0}^{\omega} r_{2}(t) \mathrm{d} t \leq 2 \omega r_{2}^{M} .
\end{aligned}
$$


12 Positive periodic solution of a cooperative model

That is,

$$
\begin{aligned}
& \int_{0}^{\omega}\left|\dot{z}_{1}(t)\right| \mathrm{d} t \leq 2 \omega r_{1}^{M}, \\
& \int_{0}^{\omega}\left|\dot{z}_{2}(t)\right| \mathrm{d} t \leq 2 \omega r_{2}^{M} .
\end{aligned}
$$

Let $t_{1} \in[0, \omega]$ such that $z_{1}\left(t_{1}\right)=\max _{t \in[0, \omega]}\left\{z_{1}(t)\right\}$, since $r_{1}(t)>0$; the first equation of (3.20) implies

$$
\begin{gathered}
\frac{\prod_{0 \leq t_{k}<t_{1}-\tau_{11}\left(t_{1}\right)}\left(1-\gamma_{1 k}\right) \exp \left\{z_{1}\left(t_{1}-\tau_{11}\left(t_{1}\right)\right)\right\}}{a_{1}\left(t_{1}\right)+b_{1}\left(t_{1}\right) \prod_{0 \leq t_{k}<t_{1}-\tau_{12}\left(t_{1}\right)}\left(1-\gamma_{2 k}\right) \exp \left\{z_{2}\left(t_{1}-\tau_{12}\left(t_{1}\right)\right)\right\}} \\
+c_{1}\left(t_{1}\right) \prod_{0 \leq t_{k}<t_{1}-\tau_{13}\left(t_{1}\right)}\left(1-\gamma_{1 k}\right) \exp \left\{z_{1}\left(t_{1}-\tau_{13}\left(t_{1}\right)\right)\right\}=1 ;
\end{gathered}
$$

hence,

$$
c_{1}\left(t_{1}\right) \prod_{0 \leq t_{k}<t_{1}-\tau_{13}\left(t_{1}\right)}\left(1-\gamma_{1 k}\right) \exp \left\{z_{1}\left(t_{1}-\tau_{13}\left(t_{1}\right)\right)\right\}<1
$$

moreover,

$$
\prod_{0 \leq t_{k}<t_{1}-\tau_{13}\left(t_{1}\right)}\left(1-\gamma_{1 k}\right) \exp \left\{z_{1}\left(t_{1}-\tau_{13}\left(t_{1}\right)\right)\right\}<\frac{1}{c_{1}\left(t_{1}\right)}
$$

then

$$
z_{1}\left(t_{1}-\tau_{13}\left(t_{1}\right)\right)<\ln \frac{A_{1}^{s}}{c_{1}^{m}}
$$

We denote $t_{1}-\tau_{13}\left(t_{1}\right)=t_{1}^{*}+l_{1} \omega, t_{1}^{*} \in[0, \omega]$ and $l_{1}$ is an integer, then

$$
z_{1}\left(t_{1}^{*}\right)<\ln \frac{A_{1}^{s}}{c_{1}^{m}}
$$

in view of this and (3.23), we have

$$
z_{1}(t)=z_{1}\left(t_{1}^{*}\right)+\int_{t_{1}^{*}}^{t} \dot{z}_{1}(s) \mathrm{d} s \leq z_{1}\left(t_{1}^{*}\right)+\int_{0}^{\omega}\left|\dot{z}_{1}(s)\right| \mathrm{d} s<\ln \frac{A_{1}^{s}}{c_{1}^{m}}+2 \omega r_{1}^{M}:=M_{1} .
$$


From (3.20), (3.30), and condition (i), it follows that

$$
\begin{aligned}
c_{1}\left(t_{1}\right) & \prod_{0 \leq t_{k}<t_{1}-\tau_{13}\left(t_{1}\right)}\left(1-\gamma_{1 k}\right) \exp \left\{z_{1}\left(t_{1}-\tau_{13}\left(t_{1}\right)\right)\right\} \\
= & 1-\frac{\prod_{0 \leq t_{k}<t_{1}-\tau_{11}\left(t_{1}\right)}\left(1-\gamma_{1 k}\right) \exp \left\{z_{1}\left(t_{1}-\tau_{11}\left(t_{1}\right)\right)\right\}}{a_{1}\left(t_{1}\right)+b_{1}\left(t_{1}\right) \prod_{0 \leq t_{k}<t_{1}-\tau_{12}\left(t_{1}\right)}\left(1-\gamma_{2 k}\right) \exp \left\{z_{2}\left(t_{1}-\tau_{12}\left(t_{1}\right)\right)\right\}} \\
> & \frac{a_{1}\left(t_{1}\right)-\prod_{0 \leq t_{k}<t_{1}-\tau_{11}\left(t_{1}\right)}\left(1-\gamma_{1 k}\right) \exp \left\{M_{1}\right\}}{a_{1}\left(t_{1}\right)} \\
\geq & \frac{a_{1}\left(t_{1}\right)-A_{2}^{s} \exp \left\{M_{1}\right\}}{a_{1}\left(t_{1}\right)}>0 ;
\end{aligned}
$$

hence,

$$
\prod_{0 \leq t_{k}<t_{1}-\tau_{13}\left(t_{1}\right)}\left(1-\gamma_{1 k}\right) \exp \left\{z_{1}\left(t_{1}-\tau_{13}\left(t_{1}\right)\right)\right\} \geq \frac{a_{1}\left(t_{1}\right)-A_{2}^{s} \exp \left\{M_{1}\right\}}{a_{1}\left(t_{1}\right) c_{1}\left(t_{1}\right)}
$$

or

$$
z_{1}\left(t_{1}-\tau_{13}\left(t_{1}\right)\right)>\ln \left[A_{1}^{f} \frac{a_{1}\left(t_{1}\right)-A_{2}^{s} \exp \left\{M_{1}\right\}}{a_{1}\left(t_{1}\right) c_{1}\left(t_{1}\right)}\right] .
$$

Therefore,

$$
\begin{aligned}
z_{1}\left(t_{1}^{*}\right) & >\ln \left[A_{1}^{f} \frac{a_{1}\left(t_{1}\right)-A_{2}^{s} \exp \left\{M_{1}\right\}}{a_{1}\left(t_{1}\right) c_{1}\left(t_{1}\right)}\right], \\
z_{1}(t) & =z\left(t_{1}^{*}\right)+\int_{t_{1}^{*}}^{t} \dot{z}_{1}(s) \mathrm{d} s \\
& >\ln \left[A_{1}^{f} \frac{a_{1}\left(t_{1}\right)-A_{2}^{s} \exp \left\{M_{1}\right\}}{a_{1}\left(t_{1}\right) c_{1}\left(t_{1}\right)}\right]-\int_{0}^{\omega}\left|\dot{z}_{1}(t)\right| \mathrm{d} t \\
& >\ln \left[A_{1}^{f} \frac{a_{1}\left(t_{1}\right)-A_{2}^{s} \exp \left\{M_{1}\right\}}{a_{1}\left(t_{1}\right) c_{1}\left(t_{1}\right)}\right]-2 \omega r_{1}^{M}:=M_{1}^{\prime},
\end{aligned}
$$

that is,

$$
z_{1}(t)>M_{1}^{\prime}
$$

From (3.35) and (3.30), we have

$$
\left|z_{1}(t)\right|<\max \left\{\left|M_{1}\right|,\left|M_{1}^{\prime}\right|\right\}:=H_{1} .
$$


14 Positive periodic solution of a cooperative model

Let $t_{2} \in[0, \omega]$ such that $z_{2}\left(t_{2}\right)=\max _{t \in[0, \omega]}\left\{z_{2}(t)\right\}$; since $r_{2}(t)>0$, the second equation of (3.20) implies that

$$
\begin{gathered}
\prod_{0 \leq t_{k}<t_{2}-\tau_{21}\left(t_{2}\right)}\left(1-\gamma_{2 k}\right) \exp \left\{z_{2}\left(t_{2}-\tau_{21}\left(t_{2}\right)\right)\right\} \\
a_{2}\left(t_{2}\right)+b_{2}\left(t_{2}\right) \prod_{0 \leq t_{k}<t_{2}-\tau_{22}\left(t_{2}\right)}\left(1-\gamma_{1 k}\right) \exp \left\{z_{1}\left(t_{2}-\tau_{22}\left(t_{2}\right)\right)\right\} \\
+c_{2}\left(t_{2}\right) \prod_{0 \leq t_{k}<t_{2}-\tau_{23}\left(t_{2}\right)}\left(1-\gamma_{2 k}\right) \exp \left\{z_{2}\left(t_{2}-\tau_{23}\left(t_{2}\right)\right)\right\}=1
\end{gathered}
$$

thus,

$$
c_{2}\left(t_{2}\right) \prod_{0 \leq t_{k}<t_{2}-\tau_{23}\left(t_{2}\right)}\left(1-\gamma_{2 k}\right) \exp \left\{z_{2}\left(t_{2}-\tau_{23}\left(t_{2}\right)\right)\right\}<1
$$

or

$$
\prod_{0 \leq t_{k}<t_{2}-\tau_{23}\left(t_{2}\right)}\left(1-\gamma_{2 k}\right) \exp \left\{z_{2}\left(t_{2}-\tau_{23}\left(t_{2}\right)\right)\right\}<\frac{1}{c_{2}\left(t_{2}\right)},
$$

then

$$
z_{2}\left(t_{2}-\tau_{23}\left(t_{2}\right)\right)<\ln \frac{B_{1}^{s}}{c_{2}^{m}}
$$

We denote $t_{2}-\tau_{23}\left(t_{2}\right)=t_{2}^{*}+l_{2} \omega, t_{2}^{*} \in[0, \omega]$ and $l_{2}$ is an integer, then

$$
z_{2}\left(t_{2}^{*}\right)<\ln \frac{B_{1}^{s}}{c_{2}^{m}}
$$

in view of this and (3.24), we have

$$
z_{2}(t)=z_{2}\left(t_{2}^{*}\right)+\int_{t_{2}^{*}}^{t} \dot{z}_{2}(s) \mathrm{d} s<\ln \frac{B_{1}^{s}}{c_{2}^{m}}+2 \omega r_{2}^{M}=M_{2} .
$$

It follows from (3.20), (3.42), and condition (ii) that

$$
\begin{aligned}
& c_{2}(t) \prod_{0 \leq t_{k}<t-\tau_{23}(t)}\left(1-\gamma_{2 k}\right) \exp \left\{z_{2}\left(t-\tau_{23}(t)\right)\right\} \\
& =1-\frac{\prod_{0 \leq t_{k}<t-\tau_{21}(t)}\left(1-\gamma_{2 k}\right) \exp \left\{z_{2}\left(t-\tau_{21}(t)\right)\right\}}{a_{2}(t)+b_{2}(t) \prod_{0 \leq t_{k}<t-\tau_{22}(t)}\left(1-\gamma_{1 k}\right) \exp \left\{z_{1}\left(t-\tau_{22}(t)\right)\right\}} \\
& >\frac{a_{2}\left(t_{2}\right)-\prod_{0 \leq t_{k}<t-\tau_{21}(t)}\left(1-\gamma_{2 k}\right) \exp \left\{M_{2}\right\}}{a_{2}\left(t_{2}\right)} \\
& \geq \frac{a_{2}\left(t_{2}\right)-B_{2}^{s} \exp \left\{M_{2}\right\}}{a_{2}\left(t_{2}\right)}>0
\end{aligned}
$$


hence,

$$
\prod_{0 \leq t_{k}<t-\tau_{23}(t)}\left(1-\gamma_{2 k}\right) z_{2}\left(t-\tau_{23}(t)\right)>\frac{a_{2}\left(t_{2}\right)-B_{2}^{s} \exp \left\{M_{2}\right\}}{a_{2}\left(t_{2}\right) c_{2}\left(t_{2}\right)},
$$

then

$$
z_{2}\left(t_{2}-\tau_{23}\left(t_{2}\right)\right)>\ln \left[B_{1}^{f} \frac{a_{2}^{m}-B_{2}^{s} \exp \left\{M_{2}\right\}}{a_{2}^{M} c_{2}^{M}}\right] .
$$

Therefore,

$$
z_{2}\left(t_{2}^{*}\right)>\ln \left[B_{1}^{f} \frac{a_{2}^{m}-B_{2}^{s} \exp \left\{M_{2}\right\}}{a_{2}^{M} c_{2}^{M}}\right]
$$

from this and (3.23), we obtain

$$
z_{2}(t)=z_{2}\left(t_{2}^{*}\right)+\int_{t_{2}^{*}}^{t} \dot{z}_{2}(s) \mathrm{d} s>\ln \left[B_{1}^{f} \frac{a_{2}^{m}-B_{2}^{s} \exp \left\{M_{2}\right\}}{a_{2}^{M} c_{2}^{M}}\right]-2 \omega r_{2}^{M}:=M_{2}^{\prime},
$$

that is,

$$
z_{2}(t)>M_{2}^{\prime}
$$

From (3.35) and (3.42) we have

$$
\left|z_{2}(t)\right|<\max \left\{\left|M_{2}\right|,\left|M_{2}^{\prime}\right|\right\}:=H_{2} .
$$

Denote $H=H_{1}+H_{2}+H_{0}$, clearly $H$ is independent of $\lambda$. Now we take $\Omega=\left\{\left(z_{1}(t)\right.\right.$, $\left.\left.z_{2}(t)\right)^{T} \in \mathbb{X}:\left\|\left(z_{1}, z_{2}\right)^{T}\right\|<H\right\}$. This $\Omega$ satisfied the condition (a) of Lemma 3.1. While $\left(z_{1}(t), z_{2}(t)\right)^{T} \in \partial \Omega \cap \mathbb{R}^{2},\left(z_{1}, z_{2}\right)^{T}$ is a constant vector with $\left|z_{1}\right|+\left|z_{2}\right|=H$. Then

$$
\mathrm{QN}\left[\begin{array}{l}
z_{1} \\
z_{2}
\end{array}\right]=\left[\begin{array}{l}
\frac{1}{\omega} \int_{0}^{\omega} G_{1}\left(t, z_{1}(t), z_{2}(t)\right) \mathrm{d} t \\
\frac{1}{\omega} \int_{0}^{\omega} G_{2}\left(t, z_{1}(t), z_{2}(t)\right) \mathrm{d} t
\end{array}\right] \neq 0 .
$$

Furthermore, take $J=I: \operatorname{Im} Q \rightarrow \operatorname{Ker} L$. By Lemma 3.2, we have

$$
\operatorname{deg}\left\{J Q N, \operatorname{Ker} L \cap \Omega,(0,0)^{T}\right\} \neq 0 .
$$

According to Lemma 3.1, system (3.4) has at least one $\omega$-periodic solution. As a consequence, system (1.3) has at least one positive $\omega$-periodic solution. The proof is complete.

\section{Acknowledgments}

This work is supported by the National Natural Sciences Foundation of China under Grant 10361006 and the Natural Sciences Foundation of Yunnan Province under Grant 2003A0001M. 


\section{References}

[1] D. Bainov and M. Dimitrova, Oscillation of sub-and superlinear impulsive differential equations with constant delay, Applicable Analysis 64 (1997), no. 1-2, 57-67.

[2] D. Bainov, M. Dimitrova, and A. Dishliev, Necessary and sufficient conditions for existence of nonoscillatory solutions of impulsive differential equations of second order with retarded argument, Applicable Analysis 63 (1996), no. 3-4, 287-297.

[3] L. S. Chen, Mathematical Models and Methods in Ecology, Science Press, Beijing, 1988.

[4] J. M. Cushing, Periodic time-dependent predator-prey systems, SIAM Journal on Applied Mathematics 32 (1977), no. 1, 82-95.

[5] A. Domoshnitsky and M. Drakhlin, Nonoscillation of first order impulse differential equations with delay, Journal of Mathematical Analysis and Applications 206 (1997), no. 1, 254-269.

[6] R. E. Gaines and J. L. Mawhin, Coincidence Degree, and Nonlinear Differential Equations, Springer, Berlin, 1977.

[7] K. Gopalsamy and B. G. Zhang, On delay differential equations with impulses, Journal of Mathematical Analysis and Applications 139 (1989), no. 1, 110-122.

[8] Y. Li and Y. Kuang, Periodic solutions of periodic delay Lotka-Volterra equations and systems, Journal of Mathematical Analysis and Applications 255 (2001), no. 1, 260-280.

[9] _ Periodic solutions in periodic state-dependent delay equations and population models, Proceedings of the American Mathematical Society 130 (2002), no. 5, 1345- 1353.

[10] R. M. May, Stability and Complexity in Model Ecosystems, Princeton University Press, New Jersey, 2001.

[11] G. Zhang and Z. Lu, On the nonautonomous cooperative system, Bulletin of Biomathematics 1 (1997), no. 3, 21-27.

Yongkun Li: Department of Mathematics, Yunnan University, Kunming, Yunnan 650091, China E-mail address: yklie@ynu.edu.cn

Wenya Xing: Department of Mathematics, Yunnan University, Kunming, Yunnan 650091, China E-mail address: xwy79hn@163.com 


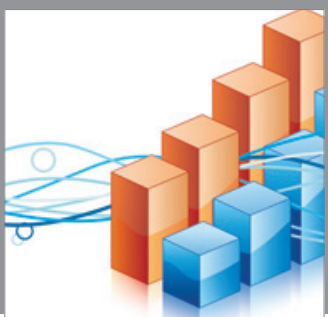

Advances in

Operations Research

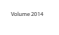

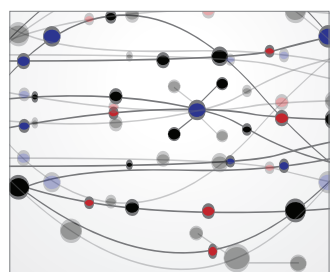

\section{The Scientific} World Journal
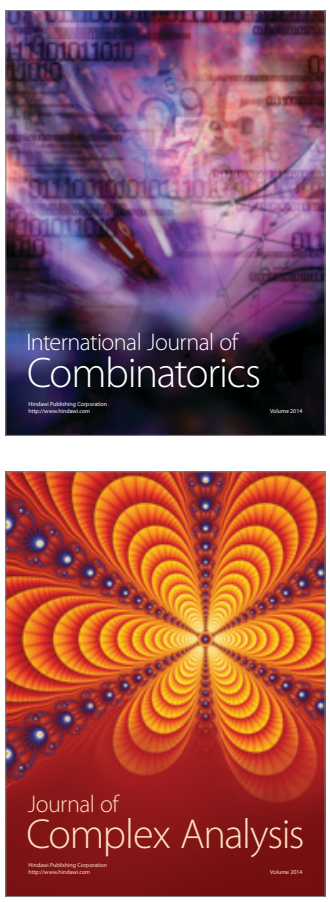

International Journal of

Mathematics and

Mathematical

Sciences
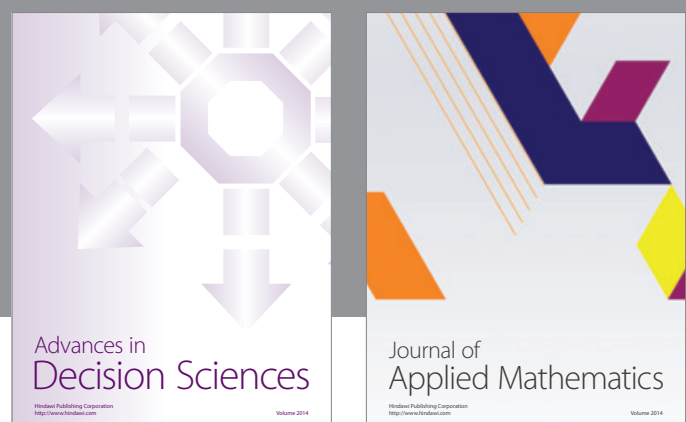

Journal of

Applied Mathematics
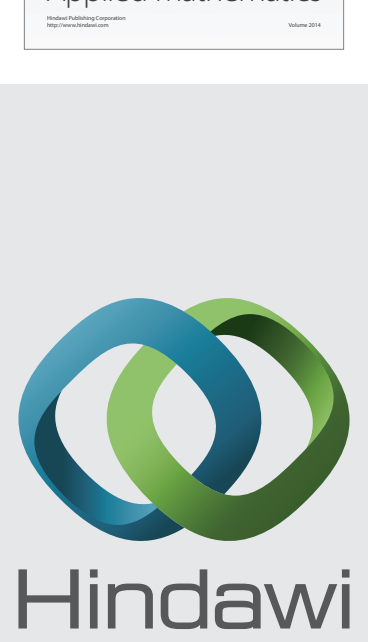

Submit your manuscripts at http://www.hindawi.com
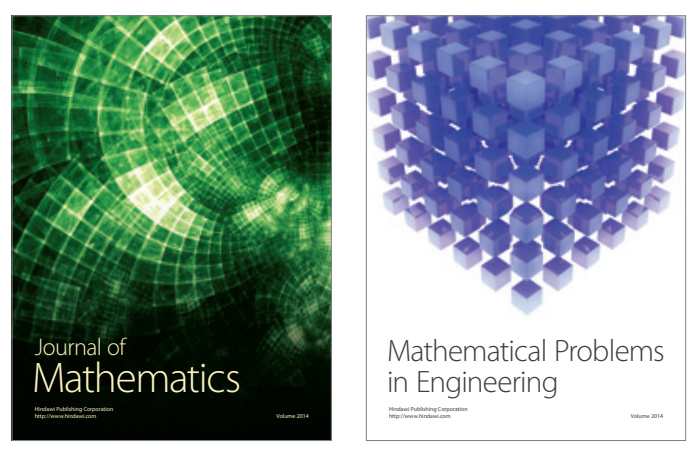

Mathematical Problems in Engineering
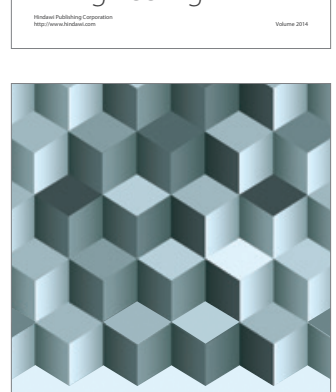

Journal of

Function Spaces
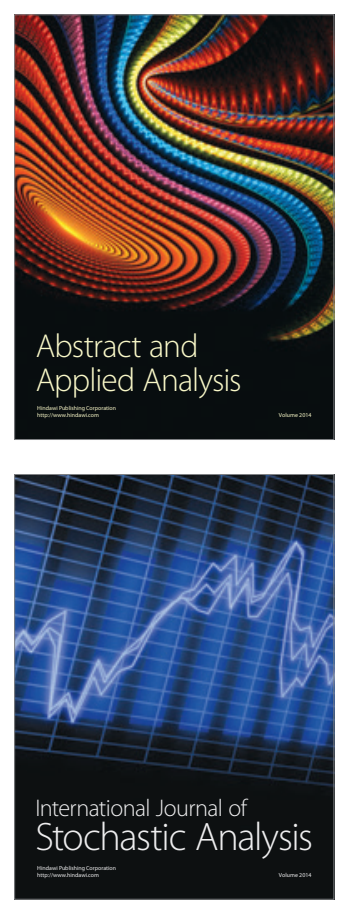

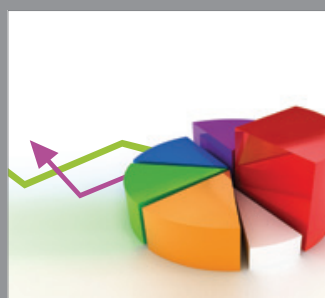

ournal of

Probability and Statistics

Promensencen
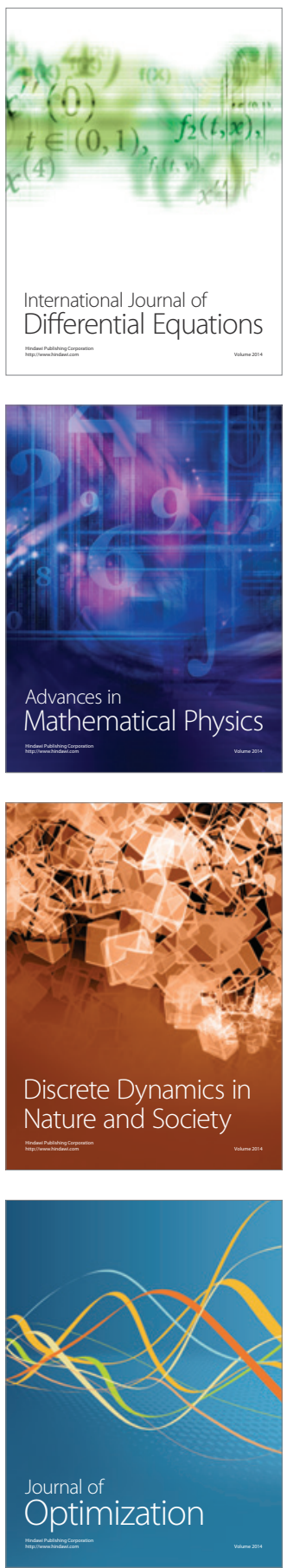